\title{
Prevalencia y pronóstico del infarto de miocardio silente en ancianos
}

Prevalence, predisposing factors, and prognosis of clinically unrecognized myocardial infarction in the elderly. Sheifer S, Gersh B, Yañez D y col. J Am Coll Cardiol 2000; 35:119-126.

\section{Objetivo}

1) Determinar la prevalencia de infarto de miocardio silente (IAM-S) en ancianos, 2) identificar factores de riesgo, 3) establecer su pronóstico en relación al infarto de miocardio reconocido (IAM-R).

\section{Diseño}

Estudio de cohorte prospectivo para análisis de mortalidad de IMS y R (Cardiovascular Health Study) y estudio transversal a partir de Los datos al ingreso a la cohorte: análisis de factores asociados con casos prevalentes* de IAM-S y R.

\section{Lugar}

Se realizó en 4 centros de referencia zonales, en Estados Unidos.

\section{Pacientes}

A partir de los integrantes de la cohorte (5888 pacientes $>65$ años, La mayoría sin enfermedad cardiovascular al ingreso) se identificaron 201 pacientes con IAM-S y 700 con IAM-R (casos preva(entes).

\section{Evaluación de factores pronósticos:}

Se definió como IAM-S aquellos pacientes que presentaban ondas Q patológicas en el electrocardiograma de inclusión a la cohorte, sin diagnóstico previo de IM referido por los pacientes. Se evaluaron los factores asociados con IAM-S. Se analizó la mortalidad de Los 3 grupos (IAM-S, IAM-R y no IAM). El análisis multivariado se ajustó por edad, sexo, factores de riesgo coronario, insuficiencia cardíaca y reconocimiento o no del IAM.

\section{Medición de resultados principales}

El punto final primario fue mortalidad total, los puntos finales secundarios: muerte cardiovascular y no cardiovascular. Las fuentes de información fueron los certificados de defunción, reportes médicos-forenses y entrevistas. El seguimiento promedio fue de 4.8 años.

\section{Resultados principales}

Al momento de la inclusión 901 pacientes presentaron IAM, de los cuales el $22.3 \%$ fueron silentes. Los factores que se asociaron de manera independiente con la presencia de IAM-S fueron ausencia de sintomas de angina e insuficiencia cardíaca. Otras variables asociadas fueron: mayor edad, mayor tensión arterial sistólica y sexo femenino. La mortalidad de los pacientes con IAM-S fue significativamente mayor que aquellos sin historia de IAM, ( $12 \%$ vs $21.4 \%$, $p<0.01)$ y similar a los pacientes con IAM-R $(25.4 \%$ vs $21.4 \%$ $p=0.24$ ). Los pacientes con IAM-S presentaron menor mortalidad cardiovascular, mayor mortalidad no cardiovascular y similar mortalidad total en relación a IAM-R. Los predictores asociados con mortalidad fueron: edad, sexo masculino, insuficiencia cardíaca, diabetes, y percepción del propio estado de salud. El reconocimiento o no del IAM no agregó información pronóstica.

\section{Conclusiones}

En la población de ancianos, los IAM-S constituyen una proporción significativa de todos Los IAM. La asociación entre IAM-S y la ausencia de angina e insuficiencia cardíaca puede reflejar alteraciones neurológicas, aunque también podría representar sesgo de diagnóstico. La mortalidad del IAM-S es similar a la de Los IAM diagnosticados.

\section{COMENTARIO}

En los ancianos, el infarto agudo de miocardio puede presentarse de maneras atípicas como por ejemplo insuficiencia cardíaca, confusión, síncope, etc. ${ }^{1}$ En estudios previos se observó una prevalencia de IAM-S de $30-43.5 \% .^{2}$, mayor que en este estudio. En estudios observacionales previos de infarto en ancianos, ${ }^{3}$ las presentaciones atípicas fueron frecuentes, aunque el electrocardiograma conservó su valor diagnóstico. A pesar de su peor pronóstico, los ancianos recibieron menos tratamiento (aunque presentan mayor reducción del riesgo absoluto). A pesar de que hay mucho publicado sobre el IAM-R, no existe suficiente información pronóstica de IAM silentes, particularmente en ancianos.

En este estudio, el diagnóstico de IAM-R se basó en el autorreporte, lo que puede introducir un sesgo de información en el diagnóstico (clasificación incorrecta). En un estudio previo, 4 el autorreporte de IAM en ancianos presentó una sensibilidad de $51 \%$ y una especificidad de $98 \%$. En relación al diagnóstico de IAM-S, se incluyeron pacientes con IAM-S prevalentes. Los casos incidentes* con mortalidad temprana y los IAM no $Q$ no están representados en este estudio, subestimando la frecuencia de IAM-S y sobrestimando u pronóstico en cuanto a sobrevida (sesgo de incidencia-prevalencia).*
En relación a los factores asociados a IMS, el análisis fue de tipo transversal, no pudiendo con este diseño establecerse relaciones causales. En relación a la ausencia de angina como factor asociado a IAM-S, podría estar relacionado con sesgo de diagnóstico (los pacientes con angina reciben mayor seguimiento y tests complementarios diagnósticos aumentando los IAM-R).

En el análisis de mortalidad, el seguimiento fue completo, a 6 años. El IAM-S presento similar pronóstico que el IAM-R, tomando en cuenta otras variables pronósticas. Los pacientes con IAM-S presentaban peores perfiles de factores de riesgo pero menor mortalidad cardiovascular (estudio no diseñado para evaluar causas específicas de muerte)

En conclusión, el IAM-S en ancianos presenta similar pronóstico que el IAM-R. El diagnóstico de IAM-S brinda información pronóstica, pero no es clara su estratificación de riesgo o si estos pacientes se benefician con diferentes tratamientos. Dada la prevalencia de IAM-S y su pronóstico, y el potencial beneficio con el tratamiento de la enfermedad coronaria, quedaría por evaluar la costoefectividad del rastreo de IAM silente en ancianos.

\section{Dr. Daniel C. Ferrante}

Cardiólogo. Clínica Suizo Argentina.

\section{Referencias}

1.Maheshwari A; Laird-Fick HS; Cannon LA Acute MI. Age-related presentations and treatment options. Geriatrics 2000 Feb;55(2):32-4, 37-40

2. Nadelman J, Frishman WH, Ooi WL, y cols. Precalence, incidence and prognosis of recognized and unrecognized myocardial infartcion in persons aged 75 years old or older: the Bronx Aging Study. Am J Cardiol 1990; 66: 533-7.

3. Herlitz J; Hartford M; Dellborg M; Karlson BW Optimal treatment after acute myocardial infarction in the elderly. Drugs Aging 1995 Mar;6(3):181-91

4. O' Donnell CJ; Glynn R.; Field TS; Averback R; Satterfield S; Friesenger GC 2nd; Taylor J0; Hennekens CH. Misclassification and under-reporting of acute myocardial infarction by elderly persons: implications for community-based observational studies and clinical trials. J Clin Epidemiol 1999 Auq:52(8):745-51 